\title{
Correction to: Reliable data dissemination for the internet of things using Harris hawks optimization
}

\author{
Ali Seyfollahi ${ }^{1}$ - Ali Ghaffari ${ }^{1}$ (D \\ Published online: 29 July 2020 \\ (C) Springer Science+Business Media, LLC, part of Springer Nature 2020
}

\section{Correction to: Peer-to-Peer Netw. Appl.} https://doi.org/10.1007/s12083-020-00933-2

The Publisher would like to correct the introduced formatting errors and presentation of equation 4 and Table 2 in the original publication.

1. Eq. 4 is typeset inappropriately by inserting unnecessary commas and may confuse the reader.

2. In Table 2, some values of the two columns are mistakenly shifted. First, the Parameters with the Experimental setup in the first row of the table must be changed. "Network area size,
Data packet size, Control packet size, Transmission layer protocol, Number of network nodes, Nodes distribution pattern, The initial energy of nodes, and Eelec" should be moved to the left column, as well as their values, move to the bottom right of the table.

The Publisher apologizes for the oversight and for any confusion it may have caused.

The original article has been corrected and the updated equation 4 and Table 2 are presented in this article.

$\vec{X}(t+1)=\left\{\begin{array}{ll}\vec{X}_{\text {rand }}(t)-r_{1}\left|\vec{X}_{\text {rand }}(t)-2 r_{2} \vec{X}(t)\right|, & q \geq 0.5 \\ \left(\vec{X}_{\text {prey }}(t)-\vec{X}_{m}(t)\right)-r_{3}\left(L B+r_{4}(U B-L B)\right), & q<0.5,\end{array} \vec{X}_{m}(t)=\frac{1}{N} \sum_{i=1}^{N} \vec{X}_{i}(t)\right.$

The online version of the original article can be found at https://doi.org/ 10.1007/s12083-020-00933-2

Ali Ghaffari

a.ghaffari@iaut.ac.ir

Ali Seyfollahi

stu.seyfollahi@iaut.ac.ir

1 Department of Computer Engineering, Tabriz Branch, Islamic Azad University, Tabriz, Iran 
Table 2 The simulation parameters

\begin{tabular}{ll}
\hline Parameter & Values \\
\hline Network area size & $100 \mathrm{~m} \times 100 \mathrm{~m}$ \\
Data packet size & 20 bytes \\
Control packet size & 2 bytes \\
Transmission layer protocol & User Datagram Protocol (UDP) \\
Number of network nodes & $100,200,300,400$ and 500 \\
Nodes distribution pattern & Random \\
The initial energy of nodes & $0.1 \mathrm{~J}$ \\
$\mathrm{E}_{\mathrm{elec}}$ & $50 \mathrm{~nJ} / \mathrm{bit}$ \\
$\varepsilon_{\mathrm{fs}}$ & $10 \mathrm{pJ} / \mathrm{bit} / \mathrm{m}^{2}$ \\
$\varepsilon_{\mathrm{mp}}$ & $0.0013 \mathrm{pJ} / \mathrm{bit} / \mathrm{m}^{4}$ \\
$\mathrm{E}_{\mathrm{DA}}$ & $\mathrm{nJ} / \mathrm{bit} / \mathrm{signal}$ \\
$\mathrm{d}_{\mathrm{toBS}}$ & Between $75 \mathrm{~m} \mathrm{to} 185 \mathrm{~m}$ \\
Number of packets forwarding operator & 300 \\
Number of control packets & 24 \\
Distance between nodes & Euclidean \\
The power of sending each node in the cluster radius & $10 \mathrm{~m}$ \\
Number of clusters & 10 and 20 \\
Number of WNs & 30 \\
The initial population of Harris' hawks & 20 \\
Number of iterations & 100 \\
\hline
\end{tabular}

Publisher's note Springer Nature remains neutral with regard to jurisdictional claims in published maps and institutional affiliations. 